\title{
Development of an in vitro drug screening assay using Schistosoma haematobium schistosomula
}

Monika Marxer ${ }^{1,2}$, Katrin Ingram ${ }^{1,2}$ and Jennifer Keiser ${ }^{1,2^{*}}$

\begin{abstract}
Background: The development of novel antischistosomal drugs is crucial, as currently no vaccine and only a single drug is available for the treatment of schistosomiasis. Fast and accurate in vitro assays are urgently needed to identify new drug candidates and research efforts should include Schistosoma haematobium. The aim of the present study was to develop a S. haematobium drug sensitivity assay based on newly transformed schistosomula (NTS).

Methods: We first undertook comparative studies on the cercarial emergence rhythms of the intermediate host snails Biomphalaria glabrata (S. mansoni) and Bulinus truncatus (S. haematobium). Two transformation methods as well as three purification methods were studied on S. haematobium cercariae in order to produce a large number of viable and clean NTS. Known antischistosomal drugs were tested in the established NTS assay in vitro. Drug effects were evaluated either microscopically or fluorometrically, using a resazurin based viability marker.

Microscopically obtained $I C_{50}$ values were compared with results obtained for S. mansoni.

Results: A circadian rhythm existed in both snail species. Infected B. truncatus snails shed less cercariae than B. glabrata during the testing period. The highest transformation rate (69\%) of S. haematobium cercariae into NTS was obtained with the vortex transformation (mechanical input) and the highest purification factor was observed using Percoll ${ }^{\circledR}$. The fluorimetric readout based on resazurin was very precise in detecting dead or/and severely damaged schistosomula.
\end{abstract}

Conclusions: With the use of viability markers such as resazurin, drug screening assays using S. haematobium NTS can be efficiently performed. However, drugs acting on the morphology and motility of S. haematobium NTS, such as metrifonate are missed. Drug sensitivity assays with NTS of both species, S. haematobium and S. mansoni, showed very similar results using known antischistosomal drugs. The S. mansoni NTS assay might be more suitable as primary screen in drug discovery efforts, which ultimately aim for a broad-spectrum antischistosomal drug as a larger number of S. mansoni NTS can be generated.

Keywords: In vitro, Chemotherapy, Schistosoma haematobium, Newly transformed schistosomula, Alamar Blue ${ }^{\circledR}$, Cercarial rhythm, Percoll ${ }^{\circledR}$, Bulinus truncatus, Biomphalaria glabrata

\section{Background}

Schistosomiasis remains one of the most prevalent helminthic infections in the world. Human schistosomiasis is caused by three main species including Schistosoma haematobium and Schistosoma mansoni. Schistosomes have a complex life cycle with snails serving as the intermediary host between the mammalian hosts. Approximately 800 million people are at risk of schistosomiasis

\footnotetext{
* Correspondence: jennifer.keiser@unibas.ch

${ }^{1}$ Department of Medical Parasitology and Infection Biology, Swiss Tropical and Public Health Institute, P.O. Box, CH-4002, Basel, Switzerland

¿University of Basel, $\mathrm{CH}-4003$, Basel, Switzerland
}

in 74 developing countries, with an estimated 200 million people currently infected. Schistosomiasis is a neglected tropical disease, however, just ranks below malaria in terms of its public health importance [1-4].

Control of schistosomiasis relies on preventive chemotherapy programs targeting the entire at risk population [5]. Up to now, praziquantel has been the drug of choice [6-8]. The drug is safe, has a broad therapeutic profile, and is cheap. However, these advantages have impeded the advances in the development of new drug candidates and vaccines $[8,9]$. In addition, praziquantel is not a 
perfect drug. It has a poor efficacy against immature worms, leading to a decreased ability of praziquantel to completely clear infections [8,9]. In addition, with the threat of drug resistance that accompanies preventive chemotherapy programs it is imperative to develop a novel antischistosomal drug. This drug should have a broad spectrum of trematocidal activity, similar to praziquantel. Given the known biological differences between S. mansoni and S. haematobium, drug screening should include testing against S. haematobium.

In the past years efforts have been made to improve drug sensitivity in vitro assays with $S$. mansoni enabling the testing of a larger number of compounds and improved readouts such as those with Alamar Blue ${ }^{\circledR}$ (a resazurin based viability marker) [10]. However, to our knowledge drug screening assays with $S$. haematobium continue to rely mainly on the adult worm.

The aim of the present work was to develop and validate an in vitro drug screening assay for S. haematobium newly transformed schistosomula (NTS). In a first step the cercarial emergence rhythms of the intermediate hosts of S. mansoni and S. haematobium, Biomphalaria glabrata and Bulinus truncatus were studied. Next, two artificial transformation methods for the production of the schistosomula were compared. In addition, the best purification method and optimal culture conditions were established by testing three different methods and several different media [11-14]. Finally, an assay based on the viability marker resazurin, which is the active component of Alamar Blue ${ }^{\circledR}$ and a redox indicator of enzyme activity, using selected compounds of known antischistosomal activity such as praziquantel, mefloquine, artesunate, metrifonate, and oxamniquine was established and the results compared to a standard motility assay using microscopical readout. Results were compared with drug activity observed microscopically against $S$. mansoni NTS.

\section{Methods}

\section{Drugs and media}

Praziquantel (Sigma Aldrich), mefloquine (kindly provided by Mepha Pharma AG, Switzerland), artesunate (kindly provided by Mepha Pharma AG, Switzerland), metrifonate (kindly provided by Bayer Animal Health, Germany), and oxamniquine (kindly provided by Q. Bickle, London School of Hygiene and Tropical Medicine) were used as antischistosomal compounds. Drugs were dissolved in dimethylsulphoxide (DMSO) (Sigma Aldrich) to obtain drug stock solutions of $10 \mathrm{mg} / \mathrm{ml}$ and then diluted into culture media. A resazurin solution was prepared by dissolving $12.5 \mathrm{mg}$ resazurin (Sigma Aldrich) in $100 \mathrm{ml}$ of $1 \mathrm{x}$ PBS. Different media such as Basch Medium 169 (prepared in our laboratories), Dulbecco's Modified Eagle's Medium (DMEM) (Gibco) and Medium 199 (Invitrogen, Carlsbad, CA) were tested and compared to obtain optimal culture conditions for the S. haematobium NTS. All test media were supplemented with $5 \%$ heat-inactivated fetal calf serum (FCS) based on the culture media protocol of Bash 1981 [12] and $200 \mathrm{U} / \mathrm{ml}$ penicillin and $200 \mu \mathrm{g} / \mathrm{ml}$ streptomycin (Sigma Aldrich).

\section{Snails and schistosomes}

Bulinus truncatus infected with S. haematobium were obtained from BEI Resources, NIAID (NIH: Schistosoma haematobium exposed Bulinus truncatus subsp. truncatus, NR-21965). S. mansoni infected B. glabrata are maintained at the Swiss Tropical and Public Health Institute in Basel. The snails had been individually infected with either 10 miracidiae per snail (B. truncatus) or 8 miracidiae per snail (B. glabrata). Snails were kept in tanks with dechlorinated tap water in a humid room simulating a 12 hour day and night cycle. First shedding of cercariae occurred 4-6 weeks post infection. Both snail species were then collected in the morning (Size of snails: $6-11 \mathrm{~mm}$, number of collected snails: approximately 70-90 of each species) and placed individually into 24 or 48 well plates $(1 \mathrm{ml}$ dechlorinated tap water/ well). Each well plate was placed under a direct 2000 lux lamp for at least 5 hours. The cercarial suspension of each B. glabrata/B. truncatus was collected and used for the mechanical and chemical transformation. For the cercarial emergence rhythm studies, B. glabrata/B. truncatus were collected $(\mathrm{n}=90)$ in the morning at 8 am and put, as described above, in dechlorinated water filled well plates $(1 \mathrm{ml} /$ well) until $3 \mathrm{pm}$. During this period the cercariae suspension of each snail was collected hourly and counted for each snail under a light microscope. The hourly collected cercariae suspension was replaced with dechlorinated tap water.

\section{Vortex transformation}

The vortex transformation was performed based on a slightly adapted protocol of Ramalho-Pinto [11]. Briefly, a cercarial suspension was placed on ice for 30-40 minutes in order to reduce parasite motility. Tubes were then centrifuged for 3 minutes at $3000 \mathrm{rpm}$. The cercarial pellet was resuspended in $2 \mathrm{ml}$ cold Hanks` Balanced Salt Solution (HBSS) containing $2 \%$ amphotericin B (Sigma Aldrich). The suspension was vigorously mixed through a pipette followed by 4 minutes vortexing in order to induce tail shedding.

This step was repeated after an incubation of the mixed tail-schistosomula suspension for 20 minutes at $37^{\circ} \mathrm{C}$. The transformation rate was calculated by counting the total number of cercariae in the HBSS suspension before transformation, and placing them in relation to the total number of schistosomula obtained after 
purification. The transformation rate was calculated for five identically performed mechanical transformations.

\section{Glucose induced transformation}

The chemical transformation was carried out as described previously [11]. The cercariae suspension was cooled on ice for 30 minutes to reduce parasite motility. After 2 minutes of centrifuging at $2000 \mathrm{rpm}$, the cercariae suspension was resuspended in $4 \mathrm{ml}$ of $5 \%$ glucose and incubated for 10 minutes in a $30^{\circ} \mathrm{C}$ waterbath. The tails were removed from the bodies using the ice purification method (see next paragraph). The transformation rate was calculated as described above and was calculated for five identically performed chemical transformations.

\section{Purification and culture of schistosomula}

The separation of the bodies from the tails by centrifugation on a $70 \%$ Percoll $^{\circledR}$ gradient (polyvinylpyrolidonecoated colloidal silica particles) was based on the method of Lazdins et al. [13]. $71.7 \mathrm{ml}$ of Percoll ${ }^{\circledR}$ (Sigma Aldrich, starting density $1.13 \mathrm{~g} / \mathrm{ml}$ ) was diluted directly in $10 \mathrm{ml} 1.5 \mathrm{M} \mathrm{NaCl}$ and filled up to $100 \mathrm{ml}$ with distilled $\mathrm{H}_{2} \mathrm{O}$ to obtain a final working solution. $10 \mathrm{ml}$ of the working Percoll ${ }^{\circledR}$ solution was layered on the bottom of a $50 \mathrm{ml}$ Falcon tube. The gradient was topped by carefully adding $5 \mathrm{ml}$ of the transformed schistosomula suspension. The tube was then centrifuged for $10 \mathrm{~min}$ utes at $2000 \mathrm{rpm}$ and $5^{\circ} \mathrm{C}$. After centrifugation, $5 \mathrm{ml}$ of the sample was collected from the bottom of the tube after perforation using a heated 16-gauge needle. The collected fraction was then diluted in $7 \mathrm{ml}$ of Medium 199 and centrifuged for another 3 minutes at $3000 \mathrm{rpm}$. The schistosomula pellet was resuspended in $1 \mathrm{ml}$ of fresh, warm supplemented Medium 199.

Another purification method of the schistosomula was based on a simple and easy swirling technique according to F. Lewis et al. [14]. The schistosomula suspension was placed on a Petri dish and sufficient warm Medium 199 added so that the bottom of the Petri dish was completely covered. By swirling the dish gently, all schistosomula accumulated in the center and could be transferred into Falcon tubes. The swirling and collecting step was repeated until schistosomules were no longer present in the center of the dish (approximately 4-5 times).

In addition, the ice method was tested for purification. $7 \mathrm{ml}$ of cold HBSS was added to the schistosomula suspension and cooled on ice for 7 minutes. The supernatant was decanted, and the pellet resuspended again in $7 \mathrm{ml}$ of cold HBSS. This step was repeated three times. The pellet that contained the recovered schistosomula was then resuspended in preheated $\left(37^{\circ} \mathrm{C}\right)$ supplemented Medium 199.
After the experiment the number of heads and tails were counted using a sample of $50 \mu \mathrm{l}$. The ratio was expressed as purification factor. Every method was performed three times identically and the mean purification factor was calculated.

After successfully transforming cercariae into schistosomula and purifying them, the NTS were incubated for at least 12 hours in supplemented Medium 199 at an atmosphere of $37^{\circ} \mathrm{C}$ with $5 \% \mathrm{CO}_{2}$ until usage to assure complete transformation [10].

Different media, namely supplemented Basch Medium 169, Dulbecco's Modified Eagle's Medium and Medium 199 were tested and compared to obtain optimal culture conditions for the $S$. haematobium NTS. Viability of NTS (150-200 per media) was assessed based on their morphology and motility using a viability scale ranging from 3 (normal activity, no morphological changes) to 0 (dead), as described previously using increments of 0.5 [15].

\section{In vitro S. haematobium drug assay with microscopical or fluorimetric readout}

NTS were obtained by mechanical transformation of S. haematobium cercariae as described above. The schistosomula suspension was adjusted to a concentration of 2 NTS per $\mu \mathrm{L}$ with supplemented Medium 199. A 3-fold serial dilution was next performed vertically down a flat bottom 96-well plate, to obtain final drug concentrations of $1.1,3.3,10,30,90 \mu \mathrm{g} / \mathrm{ml}$ after adding $50 \mu \mathrm{l}$ of the adjusted NTS suspension yielding a final volume of $250 \mu \mathrm{l}$ per well [16]. Each drug concentration was tested in duplicate and performed at least three times. Live and dead schistosomula (treated with $70 \%$ ethanol) served as a positive and negative control.

For the microscopical readout, assays were evaluated under an inverted bright-field light microscope (Carl Zeiss AG, $8 \times 10$ magnification) 24, 48 and 72 hours post drug exposure.

NTS were evaluated using the viability scale as described above. $\mathrm{IC}_{50}$ values were calculated using CompuSyn software (Version 3.0.1, 2007; ComboSyn, Inc).

Furthermore, drug effects were determined with the help of resazurin, a fluorimetric marker for cell viability [17]. Assays were conducted as described above with the exception that 48 hours post drug exposure $20 \mu \mathrm{l}$ of the prepared resazurin solution was added to each well. Background fluorescence and absorbance of the drug containing medium were determined for each drug dilution. Wells without drug served as controls. After another 24 hours of incubation, fluorescence development was determined (after a total drug incubation time of 72 hours). Fluorescence was measured using a Spectramax M2 plate reader (Molecular Devices) at $530 \mathrm{~nm}$ excitation wavelength and $590 \mathrm{~nm}$ emission wavelength. 
The $\mathrm{IC}_{50}$ of each drug was calculated based on the fluorescence detection using the Softmax Pro Program (Molecular Devices).

In vitro S. mansoni drug assay with microscopical readout NTS from S. mansoni were obtained with the same vortex transformation procedure as mentioned above. The ice purification method was sufficient to separate the bodies from the tails. A lower antibiotic dose (1\% penicillin-streptomycin mix) was used for the assays, as lower contamination occurred here. Evaluation was done microscopically using the same viability scale $(0-3)$ as mentioned before.

\section{Statistical analysis}

Arithmetic Means and standard deviation were calculated using Microsoft Excel ${ }^{\circledR}$ software for cercarial shedding patterns, transformation and purification factors, fluorescent signals and evaluated $\mathrm{IC}_{50}$ values. All values were tested for normality. Student's one-sample $t$-test was used to analyze the statistical significance of differences between mean experimental and control values of the fluorescent values for each NTS drug assay. A $\mathrm{P}$-value of $<0.05$ was considered significant.

\section{Results}

\section{Cercarial emergence rhythm}

The cercarial shedding of the two snail species $B$. truncatus and B. glabrata followed a clear circadian rhythm, after a simulated 12:12 hour light dark cycle in the laboratory. There was one shedding peak a day which slightly differed in time and intensity according to the species (Figure 1). The daily emergence pattern peak for B. glabrata occurred between 11 am and $1 \mathrm{pm}$, whereas for $B$. truncatus the peak occurred a little earlier between $10 \mathrm{am}$ and $11.30 \mathrm{am}$. The total number of cercariae shed was 2.7-fold higher for S. mansoni infected Biomphalaria snails than for S. haematobium infected Bulinus snails.

\section{Transformation and purification}

The highest transformation rate (mean of 69\%) was observed for the vortex transformation. The previously described protocol for the vortex transformation [11] had to be slightly modified, such as increasing the number of pipetting (40x), increasing the vortex time (4 minutes) and using HBSS medium supplemented with $2 \%$ amphotericin $\mathrm{B}$ in order to achieve a continuously high transformation rate. Since high transformation rates were achieved with supplemented HBSS no other media were tested during the transformation procedure. A transformation rate of only $34 \%$ (mean value) was obtained with the chemical transformation. Both transformation methods were easy to perform.

In order to obtain the best method for purifying the NTS from the tails shed after transformation, a purification factor was calculated for three purification methods $\left(\right.$ Percoll $^{\circledR}$, ice and swirling method). The best purification factor was observed for Percoll ${ }^{\circledR}$ with 24.4 \pm 11.4 , followed by the swirling method with $11.7 \pm 3.2$ and the ice method presenting a mean purification factor of $3 \pm 1.7$.

\section{Optimal culture conditions for S. haematobium schistosomula}

All media for $S$. haematobium were supplemented with $200 \mathrm{U} / \mathrm{ml}$ penicillin and $200 \mu \mathrm{g} / \mathrm{ml}$ streptomycin. This was the ideal concentration of antibiotics to get rid of bacterial contamination that occurred from B. truncatus snail excrement, which could not be eliminated during sedimentation in the first vortex transformation steps. Supplemented Medium 199 turned out to be the most

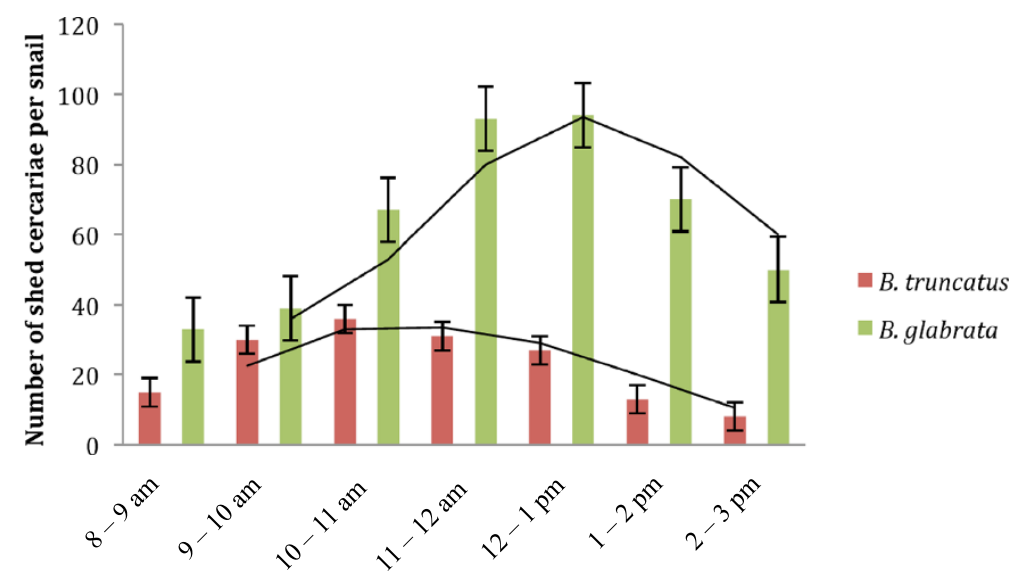

Figure 1 Circadian rhythm of cercariae released from S. haematobium infected B. truncatus and S. mansoni infected B. glabrata snails. Mean values of $(n=3)$ performed experiments are shown, bars indicate standard deviations. 
suitable medium for the incubation of schistosomula obtained by transformation in supplemented HBSS. The parasites remained viable for $120 \mathrm{~h}$ with an average viability value of 2.5 . On the other hand, all schistosomula had died by 72 hours in Basch medium and by 144 hours in DMEM (Figure 2).

\section{Evaluation of resazurin as potential fluorimetric marker for the S. haematobium NTS in vitro assay}

In an initial experiment the relationship between the fluorescence signal of the converted resazurin and schistosomula numbers (0-250 NTS tested) was studied. A linear relationship $\left(\mathrm{R}^{2}=0.95\right)$ between fluorescence development and NTS number was observed within a range of 25-200 NTS per well, as depicted in Figure 3. No increase in the fluorescence signal was observed using 250 schistosomula per well and above. Dead schistosomula and medium did not show any significant background signals.

\section{In vitro S. haematobium NTS drug assay using microscopical readout compared with fluorimetric readout}

As seen in Table 1, mefloquine showed the highest activity $\left(\mathrm{IC}_{50}\right.$ value of $\left.0.5 \mu \mathrm{M}\right)$ followed by artesunate (1.4 $\mu \mathrm{M})$, praziquantel $(1.5 \mu \mathrm{M})$ and metrifonate $(1.6 \mu \mathrm{M})$ against $S$. haematobium NTS using microscopical readout. For oxamniquine only low to moderate antischistosomal effects were observed against the schistosomular stage $\left(\mathrm{IC}_{50}: 26.5 \mu \mathrm{M}\right)$. Severe effects on the morphology of S. haematobium NTS were observed 24, 48 and 72 hours post exposure with praziquantel, mefloquine and artesunate. Concentrations of $90 \mu \mathrm{g} / \mathrm{ml}$ praziquantel and mefloquine killed the parasite within 48 and 24 hours, respectively. Both drugs caused strong morphological changes such as severe deformation and increased granularity. Metrifonate killed all the parasites, after 24 hours drug exposure with $90 \mu \mathrm{g} / \mathrm{ml}$ and after
72 hours at a concentration of $30 \mu \mathrm{g} / \mathrm{ml}$. At lower concentrations such as $1.1 \mu \mathrm{g} / \mathrm{ml}, 3.3 \mu \mathrm{g} / \mathrm{ml}$ and $10 \mu \mathrm{g} / \mathrm{ml}$, motility was reduced until hardly any movement could be observed after 48 and 72 hours. Oxamniquine treatment caused only slight morphological changes such as rounding but resulted in strongly reduced motility.

In a next step, $\mathrm{IC}_{50}$ values were determined in this assay using resazurin as viability marker. Praziquantel revealed the highest activity with an $\mathrm{IC}_{50}$ below $3.5 \mu \mathrm{M}$ $(\mathrm{P}<0.05)$ (Table 1$)$. Mefloquine $(6.4 \mu \mathrm{M})$ and artesunate $(7.4 \mu \mathrm{M})$ showed similar promising activities whereas oxamniquine $(>322.2 \mu \mathrm{M})$ and metrifonate $(348.3 \mu \mathrm{M})$ were characterized by a low activity in this assay (all $\mathrm{P}<0.05)$.

The $\mathrm{IC}_{50}$ values obtained for praziquantel, mefloquine and artesunate against $S$. haematobium NTS presented within similar ranges when evaluated microscopically or fluorimetrically. Oxamniquine lacked an antischistosomal effect according to the fluorimetric readout $\left(\mathrm{IC}_{50}>322.2 \mu \mathrm{M}\right)$ while a moderate activity was observed when the effects of the drug were assessed microscopically $(26.5 \mu \mathrm{M})$. To note, great differences were also observed between the readouts for metrifonate (348.3 $\mu \mathrm{M}$ versus $1.6 \mu \mathrm{M})$.

\section{Comparison of $\mathrm{IC}_{50}$ values of S. haematobium and S. mansoni NTS}

Using microscopy, very similar $\mathrm{IC}_{50}$ values of the 5 standard drugs were calculated for S. haematobium and S. mansoni NTS (Table 1).

\section{Discussion}

S. haematobium is a neglected parasite that still affects several million people per annum [1-4]. There is currently one drug available as the core treatment of schistosomiasis and it is worrying that low cure rates with praziquantel have already been reported in several endemic countries for S. mansoni [18]. To our knowledge,

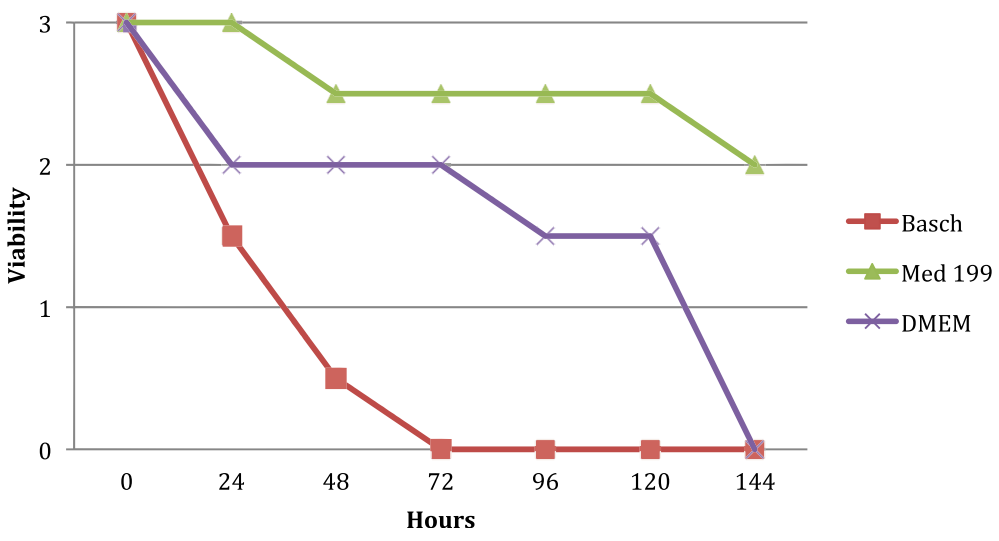

Figure 2 Survival time of S. haematobium NTS (150-200 NTS per well) in different culture media. 


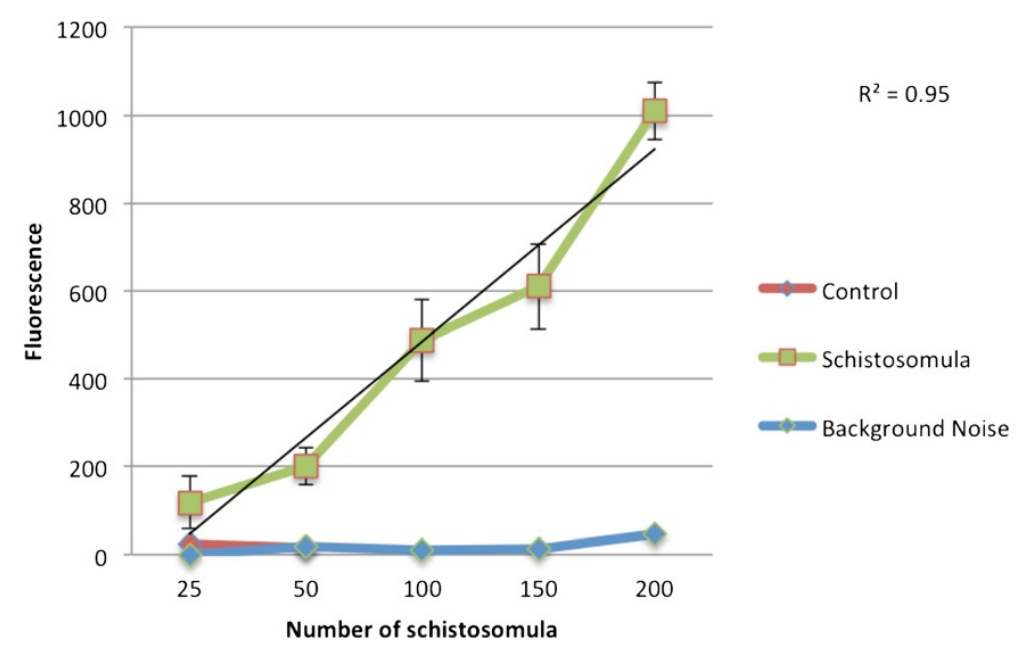

Figure 3 Relationship between fluorescence signal and number of schistosomula (25-250 schistosomula per well) in the resazurin based assay. Figure shows an average curve of four replicates, bars indicate standard deviations of the performed experiments. Fluorescence was measured after 24 hours of incubation. The fluorescence decreased with schistosomula concentrations above 200 per well. Linear regression is shown for data of 25-200 NTS.

we have for the first time established an in vitro drug sensitivity assay using S. haematobium NTS and additionally evaluated resazurin as a viability marker in this assay.

We first focused on the intermediate host snails and observed that the cercarial emergence rhythm of $B$. glabrata infected with S. mansoni as well as B. truncatus with $S$. haematobium showed one distinct peak for each species. It is important to state that these observations are based on long term laboratory isolates and the cercarial output of the imported Bulinus snails could also be influenced by the transportation between laboratories

Table 1 Comparison of the $I_{50}$ values of tested drugs for S. haematobium and S. mansoni schistosomula at the 72 hours time point evaluated microscopically and for $S$. haematobium NTS additionally fluorimetrically with resazurin

\begin{tabular}{|c|c|c|c|}
\hline \multirow[t]{2}{*}{ Technique } & \multirow[t]{2}{*}{ Drug } & \multicolumn{2}{|c|}{ IC 50 values $(\mu \mathrm{M})($ mean $\pm S D)$} \\
\hline & & S. haematobium & S. mansoni \\
\hline \multirow[t]{5}{*}{ Microscopy } & Praziquantel & $1.5 \pm 1.1$ & $0.7 \pm 0.1$ \\
\hline & Mefloquine & $0.5 \pm 0.2$ & $0.7 \pm 0.1$ \\
\hline & Artesunate & $1.4 \pm 0.1$ & $1.5 \pm 0.6$ \\
\hline & Metrifonate & $1.6 \pm 0.0$ & $0.8 \pm 0.1$ \\
\hline & Oxamniquine & $26.5 \pm 3.4$ & $11.8 \pm 0.0$ \\
\hline \multirow[t]{5}{*}{ Resazurin } & Praziquantel & $<3.5$ & ND \\
\hline & Mefloquine & 6.4 & ND \\
\hline & Artesunate & 7.4 & ND \\
\hline & Metrifonate & 348.3 & ND \\
\hline & Oxamniquine & $>322.2$ & ND \\
\hline
\end{tabular}

$\mathrm{IC}_{50}$ values are calculated from three replicates at a concentration range of 1.1-90 $\mu \mathrm{g} / \mathrm{ml}$. ND: Not determined.
( $\sim 3$ days of transport). However, the daily emergence pattern peak for B. glabrata occurred between 11 am and $1 \mathrm{pm}$, whereas for $B$. truncatus the peak occurred a little earlier between $10 \mathrm{am}$ and $11.30 \mathrm{am}$. These results underline the fact that infected B. glabrata and $B$. truncatus snails show a distinct cercarial chronobiology $[19,20]$. It has been shown that B. glabrata shed approximately 2.7 times the number of cercariae per day (mean of 446 cercariae per snail) compared to $B$. truncatus (mean of 160 cercariae per snail), despite B. truncatus being exposed to a greater number of miracidia (10 per snail) than B. glabrata (8 per snail). We did not observe any influence of size or appearance of the snails such as calcareous deposit (calcification strips on the snail's shell) on the daily cercarial shedding in B. truncatus snails. Surprisingly, these findings contradict a previous report [21] that had shown that cercarial output is related to the size of snail, meaning larger snails (9-10 $\mathrm{mm}$ diameter) shed much more cercariae than smaller sized snails $(5-6 \mathrm{~mm})$.

The mechanical transformation as described by Ramalho-Pinto et al. for S. mansoni was successfully tested on S. haematobium and yielded a transformation rate of $69 \%$ [11]. Overall both transformation methods, mechanical and chemical turned out to be very applicable on S. haematobium cercariae and were simple to perform. It is interesting to note that the chemical stimulation had a much lower transformation rate (30-41\%) than the mechanical stimulation (59-87\%). Salafsky et al. [22] reported that chemically transformed cercariae lost their osmoregulation ability at a much higher rate than during any other transformation method. Furthermore, Ramalho-Pinto et al. [11] 
observed that tail-less cercariae have increased water sensitivity as compared to fully intact cercariae. These findings might explain the reason for the lower transformation rate in chemical stimulation.

We made the observation that increasing the time of vortexing resulted in decreased fitness of the parasites over time (data not shown). For this reason, we did not further increase the vortex time. We also did not use the syringe method, which might be worthwhile testing in future studies to achieve even higher transformation rates.

After successfully transforming cercariae, schistosomula had to be purified from the tails shed and fully intact cercariae. Unfortunately, the ice method described by Fred Lewis [14] turned out to be less successful with S. haematobium and thus two other purification methods were tested, namely the swirling method and Percoll $^{\circledR}$ method, which were previously described in the literature $[13,14]$. Both of these methods successfully separated tails from the schistosomula. Percoll ${ }^{\circledR}$ turned out to be twice as effective as the swirling method. The silica colloid gradient was non-toxic and did not affect any assay procedures [13]. Schistosomula isolated by centrifugation on Percoll ${ }^{\circledR}$ did not show any loss in viability, as evaluated by morphological examination. However, it must be emphasized that using Percoll ${ }^{\circledR}$ for the purification is an expensive method compared to the swirling method. Nevertheless, taking all advantages of the gradient together, Percoll ${ }^{\circledR}$ was used for all further transformation and purification experiments performed.

Drug sensitivity screenings were performed on newly transformed $S$. haematobium schistosomula, derived from successful vortex transformations, first with the classical microscopical readout. Note that, the assessment of the parasite viability microscopically in vitro is based on two parameters: regular or lack of movement of larvae (motility) and morphological changes such as granularity and shape alterations [22]. However, microscopic assessment is subjective, slow, labor intensive and requires experience $[23,24]$. For this reason, it is crucial to find other evaluation and screening methods based not only on microscopic examination. In our study the widely used viability indicator resazurin, the active component of Alamar Blue ${ }^{\circledR}$, was evaluated.

In our preliminary studies it could be shown that a linear correlation exists between the fluorescence signal and viable transformed schistosomula. Fluorescence values per well increased proportionally to the number of schistosomula up to the amount of 200 schistosomula. The use of more than 200 schistosomula resulted in decreased fluorescence values. This might be due to crowding effects in the well.

A correlation is observed between microscopic and fluorometric readout treating S. haematobium with artesunate, praziquantel and mefloquine. All three drugs showed clear dose response relationships (decrease in viability with increasing concentrations) in both the microscopic and fluorometric readout. However, the determined $\mathrm{IC}_{50}$ values in the resazurin based assay presented a slightly higher range compared to values calculated based on microscopy. This indicates that the sensitivity of resazurin as an indicator of metabolic activity, shows a higher sensitivity on the detection of viability than the motility observations via the microscope. Furthermore, it is important to mention that in particular for the microscopic results for several of the tested drugs, the lowest concentrations tested were in the range of the calculated $\mathrm{IC}_{50}$ values (e.g. mefloquine), and lower concentrations were not tested in the present work resulting in extrapolated $\mathrm{IC}_{50}$ values. On the other hand, important morphological criteria are taken into account within the microscopic approach, which might be missed using fluorimetric viability markers. Interestingly, the fluorometric readout regarding oxamniquine and metrifonate treatment showed that NTS were still alive and metabolically functioning but the observation of the treated parasites via the microscope showed strong changes on motility and slight morphological changes of the treated schistosomula. Summarizing, one can state that a fluorimetric drug assay with S. haematobium NTS based on resazurin is efficient in detecting severely damaged respectively dead schistosomula, as already described for S. mansoni NTS [10]. Unfortunately, the assay cannot detect drug effects on the motility of the worms or drugs with slight morphological damages as demonstrated for oxamniquine and metrifonate.

In our panel of known antischistosomal drugs, metrifonate and oxamniquine were included though both drugs have been replaced by praziquantel and are not used any longer [6]. Interestingly, we observed a good in vitro activity of metrifonate, a drug recommended for treating infections with $S$. haematobium, on S. mansoni NTS, which is in line with a previous study using adult worms [25]. Oxamniquine (used for the treatment of $S$. mansoni infections) showed moderate activity against S. haematobium and S. mansoni NTS, a finding, which is in contrast to previous results using in vitro cultures with adult worms [26]. S. haematobium adults were not affected by oxamniquine, while a moderate activity was observed against S. mansoni adult worms $[15,26]$. These varying drug sensitivities between the schistosomular and adult stage might possibly be explained with differences in drug activating enzymes, the main target of oxamniquine [26].

\section{Conclusions}

We have successfully developed an in vitro assay based on S. haematobium NTS. Using mechanical stress to transform cercariae into schistosomula instead of 
working with adult worms from mice and hamsters reduces the use of animals and thus stands in accordance with the 3Rs animal protection principles [9]. It should be highlighted that although schistosomula have great advantages when used for viability assays, it is crucial not to neglect adult schistosomes, as previously mentioned, differing susceptibilities on different parasite stages are not uncommon. Nonetheless schistosomula serve as an important starting point for the assessment of new drug candidates since it allows carrying out medium to high-throughput screenings.

The resazurin-based assay has proven to be effective, simple and possibly useful for large screening of drugs against $S$. haematobium NTS, though compounds acting solely on the motility or morphology of the worms (metrifonate, oxamniquine) might be missed.

However, it must be mentioned that working with $S$. haematobium schistosomula respectively, B. truncatus snails was more time consuming than working with $S$. mansoni/ B. glabrata. In addition to that, drug sensitivity screening for both species showed very similar results. Nevertheless, S. haematobium must not be neglected despite its more laborious work. S. haematobium infections still occur in most parts of Africa and are a major problem in developing countries [27]. For this reason, it is crucial to continue working with both species, possibly with $S$. mansoni first followed by testing of active compounds against $S$. haematobium.

\section{Competing interests}

The authors declare that they have no competing interest.

\section{Authors' contributions}

$\mathrm{MM}, \mathrm{KI}$ and $\mathrm{JK}$ designed the studies. MM carried out the experiments and wrote the first draft of the manuscript. Kl and $\mathrm{KJ}$ revised the manuscript. All authors read and approved the final version of the manuscript.

\section{Acknowledgments}

The following animals were kindly provided by BEl Resources, NIAID, NIH: Schistosoma haematobium Exposed Bulinus truncatus subsp. truncatus. We are grateful to Swiss National Science Foundation (project no. PPOOA3-114941 and PPOOP3_135170 to JK) and the Scientific \& Technological Cooperation Programme Switzerland-Russia for financial support.

Received: 25 June 2012 Accepted: 2 August 2012

Published: 9 August 2012

\section{References}

1. Manson-Bahr PEC, Bell DR: Manson's Tropical Diseases. Bailliere Tindall. 22nd edition; 2008.

2. Steinmann P, Keiser J, Bos R, Tanner M, Utzinger J: Schistosomiasis and water resources development: systematic review, meta-analysis, and estimates of people at risk. Lancet Infect Dis 2006, 6:411-425.

3. WHO: The World Health Report 2003: shaping the future. Geneva: World Health Organization; 2003.

4. Gryseels B, Polman K, Clerinx J, Kestens L: Human schistosomiasis. Lancet 2006, 368:1106-1118.

5. Montresor A, Gabrielli AF, Chitsulo L, Ichimori K, Mariotti S, Engels D, Savioli L: Preventive chemotherapy and the fight against neglected tropical diseases. Expert Rev Anti Infect Ther 2012, 10:237-242.
6. Utzinger J, Keiser J: Schistosomiasis and soil-transmitted helminthiasis: common drugs for treatment and control. Expert Opin Pharmacother 2004, 5:263-285.

7. Chitsulo L, Engels D, Montresor A, Savioli L: The global status of schistosomiasis and its control. Acta Trop 2000, 77:41-51.

8. Caffrey CR: Chemotherapy of schistosomiasis: present and future. Curr Opin Chem Biol 2007, 11:433-439.

9. Keiser J: In vitro and in vivo trematode models for chemotherapeutic studies. Parasitology 2010, 137:589-603.

10. Bickle QD, Mansour NR: Comparison of microscopy and Alamar Blue reduction in a larval based assay for schistosome drug screening. PLOS Negl Trop Dis 2010, 4:e795.

11. Ramalho-Pinto FJ, Gazinzinelli G, Howells RE, Mota-Santos TA, Figueiredo EA, Pellegrino J: Schistosoma mansoni: defined system for stepwise transformation of cercaria to schistosomule in vitro. Exp Parasitol 1974 36:360-372.

12. Bash PF: Cultivation of Schistosoma mansoni in vitro. I. Establishment of cultures from cercariae and development until pairing. J Parasitol 1981, 67:179-185.

13. Lazdins JK, Sher A, Stein MJ, David JR: Schistosoma mansoni: rapid isolation and purification of schistosomula of different developmental stages by centrifugation on discontinuous density gradients of Percoll. Exp Parasitol 1982, 53:39-44.

14. Lewis F: Schistosomiasis. Curr Protoc Immunol 1999, 19:1.1-1.28.

15. Manneck T, Braissant $O$, Haggenmüller $Y$, Keiser J: Isothermal microcalorimetry to study drugs against Schistosoma mansoni. J Clin Microbiol 2011, 49:1217-1225.

16. Ingram K, Ellis W, Keiser J: Antischistosomal activities of mefloquinerelated arylmethanols. Antimicrob Agents Chemother 2012, 56:3207-3215.

17. O'Brien J, Wilson I, Orton T, Pognan F: Investigation of the Alamar Blue (resazurin) fluorescent dye for the assessment of mammalian cell cytotoxicity. Eur J Biochem 2000, 267:5421-5426.

18. Doenhoff MJ, Cioli D, Utzinger J: Praziquantel: mechanisms of action, resistance and new derivatives for schistosomiasis. Curr Opin Infect Dis 2008, 21:659-667.

19. Théron A, Mouahid G, Moné H: Schistosoma mansoni: cercarial shedding patterns from a mixed infection of Biomphalaria glabrata with two (early and late) chronobiological variants. J Parasitol Res 1997, 83:356-358.

20. Théron A, Pages JR: Analysis and comparison of cercarial emergence rhythms of Schistosoma haematobium, S. intercalatum, S. bovis and their hybrid progeny. Int J Parasitol 1990, 20:193-197.

21. Dawood IK, Chu KY: Cercarial production from Biomphalaria alexandrina infected with Schistosoma mansoni. Bull World Health Organ 1970, 42:569-574.

22. Salafsky B, Fusco AC, Whitley K, Nowicki D, Ellenberger B: Schistosoma mansoni: analysis of cercarial transformation methods. Exp Parasitol 1988, 67:116-127.

23. Hoffmann KF, Peak E: Cross-disciplinary approaches for measuring parasitic helminth viability and phenotype. An Acad Bras Cienc 2011, 83:649-662.

24. Ndao M: Diagnosis of Parasitic Diseases: Old and new approaches. Interdiscip Perspect Infect Dis 2009, 2009:278246.

25. Denham DA, Holdsworth RJ: The effect of metrifonate in vitro on Schistosoma haematobium and S. mansoni adults. Trans R Soc Trop Med Hyg 1971, 65:696.

26. Pica-Mattoccia L, Novi A, Cioli D: Enzymatic basis for the lack of oxamniquine activity in Schistosoma haematobium infections. Parasitol Res 1997, 83:687-689.

27. Rollinson D: A wake up call for urinary schistosomiasis: reconciling research effort with public health importance. Parasitology 2009, 136:1593-1610.

\section{doi:10.1186/1756-3305-5-165}

Cite this article as: Marxer et al:: Development of an in vitro drug screening assay using Schistosoma haematobium schistosomula. Parasites \& Vectors 2012 5:165. 